\title{
Adequação do manguito durante a medida da pressão arterial: uma revisão integrativa
}

\author{
Adequacy of the cuff during blood pressure measurement: an integrative review \\ Tayse Mayara de França Oliveiraa, Taciana da Costa Farias Almeidab \\ a Enfermeira graduada pela Universidade Federal de Campina Grande (UFCG), Campina Grande, PB. Brasil.

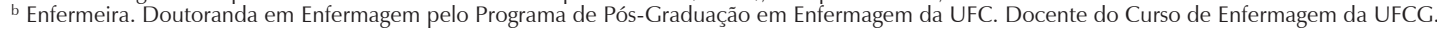

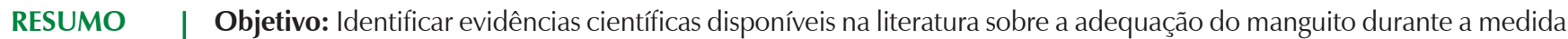
da pressão arterial.

Materiais e Métodos: Trata-se de uma revisão integrativa da literatura. As bases de dados acessadas foram: LILACS, BDENF e MEDLINE. Dos 39 artigos encontrados foram selecionados 14 por atenderem os critérios de inclusão.

Resultados: Constatou-se que $64 \%$ dos estudos foram realizados por enfermeiros e quatro (28\%) por enfermeiros e médicos. Observou-se poucos artigos publicados sobre a temática referente à adequação de manguito durante a medida da pressão arterial no Brasil nos últimos 13 anos. Lacunas no conhecimento dos profissionais envolvidos com a medida da pressão arterial e a falta de conhecimento refletiu-se também na prática, pois, apesar da oferta de diferentes manguitos e fita métrica, a totalidade dos profissionais não mediu a circunferência do braço do paciente, além de usar exclusivamente manguito com bolsa de borracha de tamanho padrão de $12 \times 23 \mathrm{~cm}$. Os estudos demonstraram que a adequação de manguito é de fundamental importância para se obter os valores corretos durante a medida da PA e para minimizar os riscos dos falsos diagnósticos.

Conclusões: A realização da medida da pressão arterial não está sendo realizada corretamente, sendo a medida da circunferência braquial um dos procedimentos menos realizados durante esta aferição.

PALAVRAS-CHAVE: Determinação da pressão arterial. Enfermagem. Pressão arterial.

Objective: To identify scientific evidence available in the literature on the adequacy of the cuff during blood pressure (BP) measurement. Materials and Methods: It is an integrative literature review. The databases accessed were LILACS, MEDLINE and BDENF. From the 39 articles found, 14 met the inclusion criteria and were selected.

Results: It was found that $64 \%$ of the studies were conducted by nurses and four (28\%) by nurses and clinicians. There were few articles published on the subject of suitability of cuff during the measurement of blood pressure in Brazil in the last 13 years. Gaps in the knowledge of the health professionals involved with measurements of blood pressure reflects in the practice. Despite the offer of different cuffs, the majority of professionals did not measure the circumference of the patient's arm. Standard size cuff with rubber bag of $12 \times 23 \mathrm{~cm}$ were the only ones used. Studies have shown that the adequacy of the cuff is extremely important to obtain the correct values for BP measurements and to minimize the risk of false diagnoses.

Conclusions: Blood pressure measurement is not often being performed adequately and the measurement of patient's arm circumference one of the least performed procedures.

KEYWORDS: Determination of blood pressure. Nursing. Blood pressure. 


\section{INTRODUÇÃO}

A medida da pressão arterial (PA) é o método diagnóstico recomendado pela Sociedade Brasileira de Hipertensão Arterial (SBH) para identificar a hipertensão arterial (HA) para o diagnóstico e prevenção de complicações cardiovasculares, deve ser realizada em toda avaliação de saúde ${ }^{1}$.

Uma das maneiras de se medir a pressão direta é por meio de técnicas invasivas (através da punção de artérias periféricas ou centrais e ligação a sistemas que possibilitem a leitura da pressão arterial média - PAM), com o uso de equipamentos sofisticados e da canulação arterial $^{2}$. Pela maior praticidade, o método indireto é realizado através do método auscultatório de Korotkof, ou através dos chamados aparelhos de pressão não-invasiva (equipamentos computadorizados que medem a PAM intermitentemente com intervalos pré-fixados e informam através de painéis digitais). Habitualmente existem três tipos de sistemas de registro do método indireto: coluna de mercúrio, eletrônico e aneroide. Os manômetros de mercúrio são considerados os mais fidedignos se comparados aos demais instrumentos. Em comum, estes métodos utilizam o manguito acoplado ao braço para realizar a medida ${ }^{3}$.

A mensuração indireta da PA é considerada um procedimento simples, não invasivo, podendo ser desenvolvido por qualquer membro da equipe multiprofissional desde que treinado. No entanto, no cotidiano das instituições de saúde, a equipe de enfermagem é a responsável pela sua realização ${ }^{4,5}$. A técnica de medida da PA realizada pelo método indireto com técnica auscultatória é um procedimento confiável e muito utilizado na prática clínica para se avaliar os níveis pressóricos ${ }^{6}$.

Diferentes fatores podem influenciar durante a medida precisa da PA. Muitos erros podem ser cometidos pelo observador (pessoa que mede a PA) devido às lacunas no conhecimento teórico, ou mesmo devido à realização do procedimento de forma mecanizada. Entretanto, o uso do manguito com tamanho errado em relação à circunferência do braço (CB) é a causa mais discutida de medida imprecisa nos últimos anos?

A largura do manguito interfere diretamente nos valores obtidos da PA, sendo a pressão subestimada quando utilizados manguitos largos e superestimada nos caso de manguitos estreitos. Para evitar que isto ocorra, a razão da circunferência braquial/largura do manguito (CB/LM) deve ser em torno de 0,40 e o comprimento do manguito deve circundar de 80 a $100 \%$ da $\mathrm{CB}^{8}$. O manguito padrão tem em média $12 \mathrm{~cm}$ de largura e $23 \mathrm{~cm}$ de comprimento, tamanho apropriado para a CB de cerca de 10 a 30\% de adultos ${ }^{9}$.
Os manguitos estreitos usados na aferição da PA em pessoas obesas superestimam o valor podendo dar um diagnóstico equivocado, pois é difícil insuflar o manguito seguindo adequadamente a técnica correta. Manguitos maiores, ao contrário, subestimam as leituras da PA em indivíduos magros, resultando em diagnósticos imprecisos e tratamentos incorretos para hipertensão ${ }^{10}$.

Apesar da grande preocupação com os erros provocados pelo uso do manguito de largura padrão (MLP), sobretudo no exterior, poucas iniciativas vêm ocorrendo para a solução do problema, especialmente aqui no Brasil ${ }^{9}$. Achados clamam para que enfermeiros, médicos e também bioengenheiros se unam na busca de medidas que ajudem a amenizar o erro e busquem diferentes soluções para um problema que já foi observado há mais de um século. Considera-se que o uso do manguito correto fornece valores de PA mais fidedignos, com maior capacidade de colaborar na previsão ou identificação precoce e prevenção da ocorrência de complicações cardiovasculares ${ }^{11}$.

A avaliação da equipe de enfermagem em quesitos básicos do conhecimento sobre a adequação do manguito durante a medida da PA é importante para que estes profissionais conheçam suas fragilidades teóricas e práticas e que assim busquem capacitação para o exercício competente de sua profissão ${ }^{12}$.

Lacunas no conhecimento dos profissionais envolvidos com a realização da técnica, especialmente em relação à adequação do manguito, assim como sua aplicabilidade, implicam em uma assistência de menor qualidade aos pacientes, riscos durante a monitorização hospitalar da PA, diagnósticos imprecisos e inadequação da terapêutica. Os resultados deste estudo são relevantes para acadêmicos de enfermagem, professores, estudiosos no tema e enfermeiros interessados em conhecer o estado da arte da produção científica sobre adequação de manguito durante a medida da PA, além de oferecer subsídios para o ensino do conteúdo nos cursos de Enfermagem.

Como forma de conduzir esta revisão foi formulada a seguinte questão direcionadora: quais são as evidências científicas disponíveis na literatura sobre a adequação de manguito durante a medida da pressão arterial? Assim este estudo teve como objetivo identificar evidências científicas disponíveis na literatura sobre a adequação do manguito durante a medida da PA.

\section{MATERIAIS E MÉTODOS}

Trata-se de uma revisão integrativa que se caracteriza como uma análise ampla da literatura sobre um assunto específico com o intuito de construir uma conclusão síntese de estudos realizados separadamente, mas que investigam problemas idênticos ou similares ${ }^{13}$. 
Para a realização deste estudo foram obedecidas as etapas expostas a seguir: identificação do tema e questões da pesquisa; amostragem ou busca na literatura; categorização dos estudos; avaliação dos estudos incluídos na revisão integrativa; interpretação dos resultados e síntese do conhecimento evidenciado nos artigos analisados ou apresentação da revisão integrativa ${ }^{14,15}$.

As fontes utilizadas foram publicações nacionais e internacionais. O levantamento bibliográfico foi realizado via internet, por meio da Biblioteca Virtual em Saúde (BVS) como fonte principal. Foram pesquisadas as seguintes base: Literatura Latino-Americana em Ciências da Saúde (LILACS), Base de dados em Enfermagem (BDENF) e Literatura Internacional em Ciências da Saúde (MEDLINE).

Para o refinamento adequado dos artigos foi definida uma amostra, obedecendo aos seguintes critérios de inclusão: estudos que foram indexados nas bases de dados a partir dos descritores controlados: determinação da pressão arterial (blood pressure determination), pressão arterial (arterial pressure) e enfermagem (nursing), independente do período de publicação; artigos publicados em português, inglês ou espanhol; artigos que retratavam procedimentos, intervenções ou diretrizes sobre determinação da PA, PA e enfermagem, estudos de natureza qualitativa, quantitativa, quali-quantitativa e revisões de literatura. Foram excluídas publicações que correspondessem a editoriais, resumos de congressos, anais, opiniões e comentários, e impossibilidade de artigo na íntegra, disponível online gratuitamente.

No mês de novembro de 2013, utilizou-se uma estratégia de busca, previamente testada, para verificar se esta iria contemplar todos os estudos pertinentes à realização da pesquisa. A busca consistiu na seleção da coleção de Literatura Científica e Técnica, onde se selecionou os descritores: determinação da pressão arterial, pressão arterial e enfermagem, e o operador booleano AND. Após a realização da estratégia de busca, procedeu-se a leitura dos títulos e dos resumos de cada publicação obtida. Posteriormente, seguiu-se a leitura na íntegra, observandose $\mathrm{o}$ atendimento aos critérios de inclusão.

Foram selecionados 39 artigos. A escolha por esta estratégia de busca deu-se porque conseguiu unir as três temáticas usadas como descritores, atendendo melhor às expectativas do estudo. Assim, os descritores foram dispostos na seguinte ordem: "determinação da pressão arterial" and "pressão arterial" and "enfermagem".

Em relação à base de dados LILACS, foram encontradas vinte e duas publicações, sendo que apenas onze atendiam aos critérios da revisão e se adequavam à temática abordada. A localização dos estudos na base de dados BDENF resultou em cinco referências; entretanto, três estudos foram excluídos, pois já haviam sido selecionados em outra base de dados (LILACS). Já na consulta à MEDLINE, a localização resultou em doze artigos, sendo dessas excluídas onze, após leitura exaustiva dos estudos, pois seis não se encontraram disponíveis online e cinco não atendiam aos critérios de inclusão do presente estudo. Assim, para esse estudo, foram obtidos um total de 14 referências.

Para a coleta de dados dos artigos incluídos na revisão integrativa, foi utilizado um instrumento elaborado por Ursi ${ }^{16}$. De acordo com a autora, esse instrumento foi submetido à validação aparente e de conteúdo. O instrumento foi adaptado para esta pesquisa, e é composto pelos seguintes itens: identificação do artigo original, instituição sede do estudo, tipo de revista científica, características metodológicas do estudo, avaliação do rigor metodológico, das intervenções mensuradas e dos resultados encontrados.

O presente estudo realizou-se de forma descritiva. A análise e síntese dos artigos obtidos nesta revisão integrativa visaram organizar e reunir o conhecimento sobre a temática a ser investigada, identificando a necessidade de investigações futuras, procurando oferecer fundamentos para a prática profissional.

\section{RESULTADOS}

Dos 14 estudos analisados, observou-se que em relação ao ano de publicação totalizaram um $(7,1 \%)$ no ano 2000 , dois $(14,2 \%)$ em 2004 , dois $(14,2 \%)$ no ano de 2006 , dois $(14,2 \%)$ em 2008 , dois $(14,2 \%)$ no ano de 2009 , dois $(14,2 \%)$ em 2012 e três $(21,4 \%)$ no ano de 2013. Em relação ao modo de aquisição, os 14 artigos foram disponibilizados online na versão completo, por acesso eletrônico às bases de dados.

Três artigos publicados no ano de 2013 (21,4\%) discutiram a avaliação da medida direta e indireta da pressão arterial, a relação da circunferência braquial com o tamanho de manguitos e o conhecimento dos profissionais da saúde. As recentes publicações demonstram uma maior preocupação dos estudiosos em relação à temática. A Tabela 1 descreve a relação dos estudos selecionados, ano de publicação e os principais resultados obtidos.

Dos 14 artigos analisados, cinco artigos publicados entre 2000 e 2006 abordam desde os métodos para se medir a PA e os erros relacionados aos falsos diagnósticos até o conhecimento dos profissionais de enfermagem sobre a largura correta do manguito; e nove artigos publicados entre 2008 e 2013 abordam desde as diretrizes brasileiras de hipertensão arterial até a relação da circunferência braquial e disponibilidade de manguitos adequados para medir a PA. 
Tabela 1 - Distribuição dos artigos segundo título, ano de publicação e resultados.

\begin{tabular}{|c|c|}
\hline & Título \\
\hline 1 & $\begin{array}{l}\text { Enfermeiros de Unidade de Terapia } \\
\text { Intensiva: avaliação sobre medida direta } \\
\text { indireta da pressão arterial }{ }^{17} \text {. }\end{array}$ \\
\hline 2 & $\begin{array}{l}\text { Relação circunferência braquial e tamanh } \\
\text { de manguitos utilizados nas Unidades } \\
\text { Básicas de Saúde de uma cidade do inte } \\
\text { paulista }^{18} \text {. }\end{array}$ \\
\hline 3 & $\begin{array}{l}\text { Análise do conhecimento teórico/prático } \\
\text { de profissionais da área da saúde sobre }\end{array}$ \\
\hline
\end{tabular}

de profissionais da área da saúde sobre medida indireta da pressão arterial ${ }^{19}$.

4 Diretrizes Brasileiras de Hipertensão arterial: realidade da enfermagem em hospital especializado ${ }^{20}$.

5 Comparação entre as técnicas de mensuração da pressão arterial em dois tempos ${ }^{21}$.

6 Medida da pressão arterial: circunferência braquial e a disponibilidade de manguitos ${ }^{7}$.

7 Uso de dispositivo automático de medida de pressão arterial em crianças entre 04 e 13 anos $^{22}$

8 Medida da pressão arterial no braço e antebraço em função do manguito ${ }^{23}$.

9 Dimensões de manguitos para crianças adolescentes: Estudo em uma cidade no Nordeste do Brasil 24

10 Registros intra-arteriais da pressão versus registros indiretos em função da largura do manguito ${ }^{21}$.

11 Fatores associados à hipertensão arteria e níveis pressóricos encontrados entre adolescentes trabalhadores ${ }^{25}$

12 O conhecimento dos profissionais da área da saúde sobre a medida da pressão arterial $^{4}$.

13 Manguitos de largura correta Levantamento em um grupo específico de crianças e adolescentes ${ }^{26}$

14 Medida da pressão arterial no paciente obeso: o método indireto com técnica auscultatória e a monitorização ambulatorial $^{27}$.

\section{Ano}

2013

Mostra a necessidade de realização urgente de atividades de educação continuada para esta amostra, visto que são profissionais que atuam diretamente com um sinal vital prioritário no cuidado ao paciente crítico.

2013 Conclui-se que em 50\% dos pacientes da amostra, o tamanho dos manguitos utilizados nas UBS para aferição de sua pressão arterial estavam adequados com a circunferência braquial, ou seja, tamanho adulto. A falta de disponibilidade de diferentes tamanhos de manguitos continua sendo um desafiante problema a ser encarado na rede pública de saúde.

2013 Concluiu-se que, de forma geral, médicos possuem sua formação, no que tange à pressão arterial voltada para a teoria, técnicos para a prática e enfermeiros encontram-se em equilíbrio entre prática e teoria. Concluiu-se que, de forma geral, médicos possuem sua formação, no que tange à pressão arterial voltada para a teoria, técnicos para a prática e enfermeiros encontram-se em equilíbrio entre prática e teoria

2012 As V Diretrizes Brasileiras de Hipertensão Arterial não são seguidas em sua plenitude pelos profissionais de enfermagem. A ampla divulgação das diretrizes, a implementação de programas de capacitação e a monitoração da técnica devem ser incentivadas.

2012 Concluiu-se que a técnica em um tempo apresentou resultados similares àqueles obtidos com a técnica em dois tempos para a amostra estudada. A técnica em um tempo não forneceu dados imprecisos para diagnósticos de hipertensão e, dessa forma, sua aplicação não privaria os pacientes de um tratamento adequado, desde que a estimativa palpatória da pressão sistólica seja incluída

2009 O manguito padrão disponível não atende às CB identificadas, resultando em registros super ou subestimados da PA.

2009 A medida de pressão arterial em crianças deve fazer parte da rotina de um exame físico e de cuidados básicos.

2009 Os achados indicam que o manguito correto diminui a hiperestimação dos registros diastólicos observada por outros autores. O avanço no conhecimento da medida no antebraço requer outros estudos.

2008 Os manguitos adequados são diferentes daqueles utilizados como padrão para crianças e adolescentes.

2006 A comparação feita entre as medidas intra-arteriais, registradas na artéria radial e medidas indiretas na braquial, em função do uso do manguito de largura correta ou do manguito de largura padrão mostrou que a medida da pressão sistólica com o manguito de largura correta indicou maior fidedignidade aos níveis arteriais registrados no monitor digital, quando comparada com o manguito de largura padrão e os níveis diastólicos registrados com o manguito de largura padrão foram menores que os registrados pelo manguito de largura correta.

2006 O uso do MLC permitiu a detecção de maior número de hipertensos e limítrofes que o uso do MLP.

2004 Foi observado que nenhum dos profissionais estudados mediu a circunferência do braço do paciente antes da medida da PA, e praticamente a totalidade fez a escolha pelo manguito com bolsa de borracha de dimensão de $12 \times 23 \mathrm{~cm}$, onde não realizaram a medida da CB do paciente.

2004 Adotar como critério para escolha dos manguitos a medida da circunferência do braço e não a idade cronológica, principalmente nos grupos de crianças e adolescentes.

2000

O principal fator de erro relacionado à medida da pressão arterial nas pessoas obesas é a relação inadequada entre o tamanho da bolsa de borracha do manguito e a circunferência do braço.
Observa-se, nos últimos cinco anos, uma maior preocupação dos estudiosos brasileiros sobre a temática medida da PA, em relação à adequação do manguito, assim como com as lacunas na execução do procedimento de medida da PA, visto que dos 14 estudos estudados, nove deles (64\%) foram publicados nos últimos cinco anos. 


\section{DISCUSSÃO}

Observou-se poucos artigos publicados sobre a temática referente à adequação de manguito durante a medida da pressão arterial, no Brasil nos últimos 13 anos. Apesar de extremamente difundida e rotineiramente utilizada, a técnica de medida da PA, principalmente pelo método auscultatório, ainda é frequentemente realizada, sem a observação das recomendações básicas para evitar erro nas aferições ${ }^{8,9}$

A preocupação das sociedades científicas com esse procedimento não é desprovida de sentido. Nos últimos cinco anos pode-se observar uma maior preocupação dos estudiosos sobre a adequação do manguito durante a medida da PA, visto que dos 14 estudos encontrados, nove (64\%) foram publicados nos últimos cinco anos. Um estudo realizado sobre a medida da PA no braço e no antebraço em função do manguito verificou as possíveis alterações dos valores da PA quando os manguitos são muito largos ou muito estreitos, concluindo que há hiperestimação dos valores quando os manguitos são mais estreitos e pela hipoestimação quando os manguitos são muito largos e os braços são finos ${ }^{23}$.

A pouca importância oferecida ao tamanho do manguito, de acordo com os estudos, é justificativa pelos profissionais pela economia de tempo que está escolha permite, ou seja, ao se medir a CB de cada paciente durante o procedimento, a técnica ficaria mais lenta, dificultando a rotina dos serviços. Um estudo ao avaliar 179 profissionais (médicos, enfermeiros e auxiliares de enfermagem) concluiu que apenas $6,7 \%$ da amostra selecionaram o manguito adequado. $\mathrm{Na}$ avaliação do conhecimento teórico destes profissionais, em todos os itens relacionados ao manguito a porcentagem de acerto foi menos da metade, refletindo na prática quando nenhum dos profissionais mediu a CB do paciente (apesar da oferta de diferentes manguitos e fita métrica no momento do procedimento) ${ }^{4}$.

A indisponibilidade dos diferentes tamanhos de manguitos é outro problema enfrentado no mercado brasileiro. É necessário e de responsabilidade das instituições prover de equipamentos em número e em condições adequadas, para garantir a obtenção de valores precisos, com atualização e educação permanente dos profissionais atuantes dessa área. Porém, tal indisponibilidade pode estar relacionada com a falta de procura pelas instituições, a qual pode estar relacionada com o desconhecimento acerca da importância de se utilizar o manguito ideal a $\mathrm{CB}^{7}$.

Torna-se urgente que os pesquisadores divulguem os conhecimentos acerca da importância da escolha do tamanho do manguito adequado durante a realização da medida da PA para os profissionais em exercício, uma vez que estes serão os disseminadores de diagnósticos de hipertensão, normotensão ou hipotensão, e os que gerarão demanda ás ofertas do mercado de manguitos, sendo assim fabricados manguitos nos diferentes tamanhos e de acordo com as diversas $\mathrm{CB}^{12}$.

Os enfermeiros brasileiros têm realizado pesquisas sobre a medida da PA e tentado atrair a atenção de especialistas para a questão da medida da PA, o qual trata-se de um problema sério e que leva a diagnósticos e tratamentos de hipertensão incorretos ${ }^{8}$. A aprendizagem da técnica de aferição da PA tem sido uma preocupação constante nas instituições de ensino de graduação, uma vez que este é um procedimento realizado rotineiramente na prática dos enfermeiros. Sendo esta uma das suas atribuições, a execução correta garante a confiabilidade e imagem de excelência de uma instituição de saúde e da profissão ${ }^{2,20}$.

Os autores dos estudos analisados afirmam que a medida da PA é o procedimento de enfermagem mais realizado no mundo. Acredita-se que esforços devam ser feitos para fornecer informação com vistas a melhorar o conhecimento de enfermagem, focando no efeito do tamanho do manguito na medida da PA. É uma questão que pode ser discutida em programas de educação permanentes, visando melhorar a consciência de enfermeiros sobre a necessidade de maior disponibilidade de instrumentos de medição, o que, consequentemente, pode melhorar a qualidade de vida dos pacientes e diminuir custos na área da saúde ${ }^{12}$.

Em um estudo sobre a medida da pressão arterial e a produção científica de enfermeiros brasileiros, observou-se que a maioria dos enfermeiros julga necessário obter mais informações a respeito da adequação de manguito durante a medida da PA e também sobre o cuidado de enfermagem a pacientes hipertensos ${ }^{9}$. Notou-se no estudo de Rabello ${ }^{4}$, que grande parte da população estudada diz ter recebido informações prévias a respeito da medição indireta da pressão arterial. No entanto muitos ainda possuem dúvidas e/ou realizam o procedimento de maneira incorreta. Da mesma forma, grande parcela diz não ter dificuldades durante a verificação da $\mathrm{PA}$, o que não condiz com as observações dos estudos realizadas durante a sua prática profissional.

Sem dúvida, a execução correta da escolha do manguito adequado durante a medida da PA é uma das atribuições do profissional de enfermagem, sua execução correta possibilita monitorar e minimizar possíveis erros associados à técnica auscultatória de verificação da pressão arterial. De uma forma geral, os estudos concluem que não é usual haver jogos de manguitos com diferentes larguras nos serviços de saúde. É provável que isto ocorra por não se considerar necessário o uso deste equipamento, que seria um custo adicional para as 
instituições. A importância do uso do manguito correto para a identificação precoce de hipertensão arterial é altamente desejável, pois implica em melhor controle da doença, diminuição das despesas com o tratamento e redução da prevalência de outras doenças para as quais ela é fator de risco ${ }^{12}$.

É preocupante o desconhecimento dos profissionais da saúde sobre a medida da PA no que diz respeito aos aspectos conceituais, técnicos, e fatores anatomo-fisiológicos. Um estudo realizado por pesquisadores espanhóis observou que dentre os profissionais da saúde, a equipe de enfermagem possui os piores resultados, mesmo quando se trata de aspectos mais técnicos do procedimento ${ }^{28}$.

A enfermagem tem se importado com a qualidade de assistência prestada e tem buscado respostas para uma assistência competente, efetiva e autônoma. Acreditase que repensar sobre os procedimentos do cotidiano da enfermagem, garantindo ao cliente qualidade na assistência, faz-se necessário e emergente. É importante que as instituições de saúde busquem medidas que visem motivar a equipe, mudar o comportamento da mesma em relação a esse procedimento, e a conscientização da importância de melhorar a qualidade da assistência de enfermagem aos pacientes hipertensos ${ }^{29}$.

Foram encontradas evidências de que a realização da medida da PA não está sendo realizada corretamente, sendo a medida da CB um dos procedimentos menos realizados durante esta aferição ${ }^{4}$. Predominaram estudos publicados nos últimos cinco anos, o que pode evidenciar uma maior preocupação dos estudiosos sobre esta temática.

Os estudos demonstraram que a adequação de manguito é de fundamental importância para se obter os valores corretos durante a medida da PA e para minimizar os riscos dos falsos diagnósticos. Lacunas no conhecimento dos profissionais envolvidos com a medida da PA também foram encontradas. A falta de conhecimento refletiu-se também na prática, pois, apesar da oferta de diferentes manguitos e fita métrica, a totalidade dos profissionais não mediu a circunferência do braço do paciente, além de usar exclusivamente manguito com bolsa de borracha de tamanho padrão de $12 \times 23 \mathrm{~cm}^{5}$.

No Brasil ainda são poucas as publicações sobre a adequação do manguito. Assim, esta temática torna-se um importante campo de exploração por parte dos pesquisadores para ampliação do conhecimento dos profissionais da saúde, especialmente os enfermeiros, para que promovam uma assistência com qualidade e com melhor embasamento técnico-científico durante a medida da PA.

Por se tratar de um assunto ainda pouco estudado na região Nordeste, aumenta-se a necessidade de se desenvolver pesquisas de campo para mostrar a realidade desta região, sobre o tema, para que, através dos resultados encontrados obtenha-se uma melhora na realização da técnica e o desempenho dos profissionais durante a medida da PA.

A divulgação dos resultados deste estudo proporcionará aos profissionais, acadêmicos e estudiosos, o conhecimento sobre a importância de se adequar corretamente o manguito durante a medida da PA, levando a mudança de práticas mecanizadas e uma assistência de maior qualidade aos pacientes com melhor monitorização da PA e adequação mais precisa da terapêutica.

Com base nos achados desta pesquisa pode-se concluir que a realização da medida da pressão arterial não está sendo realizada corretamente, sendo a medida da circunferência braquial um dos procedimentos menos realizados durante esta aferição.

\section{REFERÊNCIAS}

1. Alavarce DC, Pierin AMG. Development of educational hypermedia to teach an arterial blood pressure measurement procedure. Rev Esc Enferm USP [online]. 2011;45(4):939-44. http://dx.doi. org/10.1590/S0080-62342011000400021

2. Schmidt A, Filho AP, Maciel BC. Medida indireta da pressão arterial sistêmica. Medicina (Ribeirão Preto) [online]. 2004;37:240-5. http://dx.doi.org/10.11606/issn.2176-7262.v37i3/4p240-245

3. Galvão EL, Batista JG, Figueiredo FL, Pereira WF, Ferreira AE. Comparação entre os métodos auscultatório e oscilométrico de determinação da Pressão Arterial. Espaç Saúde 2009;10(2):27-33.

4. Rabello CCP, Pierin AMG, Mion JD. O conhecimento de profissionais da área da saúde sobre a medida da pressão arterial. Rev Esc Enferm USP [online]. 2004;38(2):127-34. http://dx.doi.org/10.1590/S008062342004000200002

5. Veiga EV, Nogueira MS, Cárnio EC, Lavrador MAS, Moraes AS, Souza LAC, Lima NKC, Nobre F. Assessment of the techniques of blood pressure measurement by health professionals. Arq Bras Cardiol [online]. 2003;80(1). http://dx.doi.org/10.1590/S0066782X2003000100008

6. Ribeiro CCM, Lamas JLT. Comparação entre as técnicas de mensuração da pressão arterial em um e em dois tempos. Rev Bras Enferm [online]. 2012;65(4):630-6. http://dx.doi.org/10.1590/ S0034-71672012000400012

7. Veiga EV, Arcuri EAM, Cloutier L, Santos JLF. Blood pressure measurement: arm circumference and cuff size availability. Rev Latino-amo Enferm [online]. 2009;17(4).

8. Pickering TG, Salão JE, Appel LJ, Falkner BE, Graves J, Colina MN, Jones DW, Kurtz T, Sheps SG, Roccella EJ. Recommendations for blood pressure measurement in humans and experimental animals: Part 1: blood pressure measurement in humans: a statement for professionals from the Subcommittee of Professional and Public Education of the American Heart Association Council on High Blood Pressure Research. Hypertension. 2005;45(1):142-61. http://dx.doi. org/10.1161/01.HYP.0000150859.47929.8e

9. Arcuri EAM, Araújo TL, Veiga EV, Oliveira SMJV, Lamas JLT, Santos JLF. Medida da pressão arterial e a produção científica de enfermeiros brasileiros. Rev Esc Enferm USP [online]. 2007 jun.;41(2):292-8. http://dx.doi.org/10.1590/S0080-62342007000200017 
10. Brasil. Ministério da Saúde. Secretaria Executiva. Subsecretaria de Planejamento e Orçamento. Sistema de Planejamento do SUS: uma construção coletiva: Plano Nacional de Saúde (PNS) 2008/20092011. Brasília (DF): Ministério da Saúde; 2010. 168 p.

11. Lamas JLT, Arcuri EAM, Brito CM, Cruz KCT. Registros intra-arteriais da pressão versus registros indiretos em função da largura do manguito. Rev Gaúcha Enferm [online]. 2006;27(4):599-609.

12. Silva SSBE, Colósimo FC, Pierin AMG. The effect of educational interventions on nursing team knowledge about arterial hypertension. Rev Esc Enferm USP [online]. 2010;44(2).

13. Polit DF, Beck CT, Hungler BP. Fundamentos de pesquisa em enfermagem: métodos, avaliação e utilização. 5a ed. Porto Alegre: Artmed; 2004.

14. Broome ME. Integrative literature reviews for the development of concepts. In Rogers BL, Knalf KA. Concept development in nursing: foundations, techniques and applications. Philadelphia: W.B. Saunders Company; 2000. p. 231-50.

15. Silveira RCCP. O cuidado de enfermagem e o cateter de Hickman: a busca de evidências [dissertação]. Ribeirão Preto (SP): Universidade de São Paulo; 2005.

16. Ursi ES. Prevenção de lesões de pele no perioperatório revisão integrativa da literatura [dissertação]. Ribeirão Preto (SP): Universidade de São Paulo; 2005.

17. Almeida TCF, Lamas JLT. Nurses of adult intensive care unit: evaluation about direct and indirect blood pressure measurement. Rev. Esc Enferm USP [online]. 2013 June;47(2):369-76. http:// dx.doi.org/10.1590/S0080-62342013000200014

18. Freitas CCQ, Pantarotto RFR, Costa LRLG. Relação circunferência braquial e tamanho de manguitos utilizados nas Unidades Básicas de Saúde de uma cidade do interior paulista. J Health Sci Inst [online]. 2013;31(3):48-52.

19. Moreira MAD, Bernardino JR. Análise do conhecimento teórico/prático de profissionais da área de saúde sobre medida indireta da pressão arterial. Biosci J [online]. 2013; 29(1): 247-54.
20. Boll LFC, Irigoyen MC, Goldmeier S. Brazilian guidelines for arterial hypertension: the reality of nursing in a specialty hospital. Acta Paul Enferm [online]. 2012;25(5):775-80. http://dx.doi.org/10.1590/ S0103-21002012000500020

21. Ribeiro CCM, Lamas JLT. Comparação entre as técnicas de mensuração da pressão arterial em um e em dois tempos. Rev Bras Enferm [online]. 2012;65(4):630-6. http://dx.doi.org/10.1590/ S0034-71672012000400012

22. Reis RS, Lamas JLT. Uso de dispositivo automático de medida de pressão arterial em crianças entre 4 e 13 anos. Rev Eletr Enf [online]. 2009;11(4):794-800.

23. Aarcuri EAM, Rosa SCD, Scanavini RM, Denzin GSC. Arm and forearm blood pressure measurements as a functionof cuff width. Acta Paul Enferm [online]. 2009;22(1):37-42.

24. Aaraújo TL, Lopes MVO, Guedes NG, Cavalcante TF, Moreira RP, Chaves ES. Cuff dimension for children and adolescents: a study in a northeastern Brazilian city. Rev Latino-am Enfermagem [online]. $2008 ; 16(5)$.

25. Spinella C, Lamas JLT. Fatores associados à hipertensão arterial e níveis pressóricos encontrados entre adolescentes trabalhadores. Rev Esc Enferm [online]. 2007;41(2).

26. Chaves EM, Gudes NG, Moreira RP, Cavalcante TF, Lima REF, Araújo TM. Manguitos de largura correta: levantamento em um grupo específico de crianças e adolescentes. Rev RENE [online]. 2004;5(2):35-40.

27. Pierin AMG, Geraldo AM. Validação dos aparelhos automáticos e semi-automáticos de medida da pressão arterial: uma revisão sobre o assunto. Rev Bras Hipertens. 2004;7(2):65-9.

28. Casti-eira MC, Gonzalez C, Rios MT, Moliner JR, Crespo J, Dominguez M. Sabemos tomar correctamente la pression arterial? Hiperten Riesgo Vasc. 2009;26(1):7-13. http://dx.doi.org/10.1016/ S1889-1837(09)70507-0

29. Cordella MP, Palotta L, Cesarino CB. Medida indireta da pressão arterial: um programa de educação continuada para a equipe de enfermagem em um hospital de ensino. Arq Ciênc Saúde [online]. 2005;12(1):21-6. 